\title{
Mixed-Model U-Shaped Assembly Line Balancing Problems with Coincidence Memetic Algorithm
}

\author{
Parames Chutima, Panuwat Olanviwatchai
}

Department of Industrial Engineering, Faculty of Engineering, Chulalongkorn University, Bangkok, Thailand

Email: parames.c@chula.ac.th

Received December $15^{\text {th }}, 2009$; revised January $8^{\text {th }}$, 2010; accepted January $25^{\text {th }}, 2010$.

\begin{abstract}
Mixed-model U-shaped assembly line balancing problems (MMUALBP) is known to be NP-hard resulting in it being nearly impossible to obtain an optimal solution for practical problems with deterministic algorithms. This paper presents a new evolutionary method called combinatorial optimisation with coincidence algorithm (COIN) being applied to Type I problems of MMUALBP in a just-in-time production system. Three objectives are simultaneously considered; minimum number workstations, minimum work relatedness, and minimum workload smoothness. The variances of COIN are also proposed, i.e. CNSGA II, and COIN-MA. COIN and its variances are tested against a well-known algorithm namely non-dominated sorting genetic algorithm II (NSGA II) and MNSGA II (a memetic version of NSGA II). Experimental results showed that COIN outperformed NSGA II. In addition, although COIN-MA uses a marginal CPU time than CNSGA II, its other performances are dominated.
\end{abstract}

Keywords: Assembly Line Balancing, Mixed-Model U-Line, JIT, COIN

\section{Introduction}

An assembly line comprises a sequence of workstations through which a predefined set of tasks are performed repeatedly on product units while they are moving along the line. It was originally developed to support mass production of single homogeneous standardised commodity to gain a competitive unit cost. Fierce competition in the current market as well as ever-changing customer requirements forces the mass production concept to become no longer attractive. Manufacturers need to redesign their production lines to accommodate mixedmodel production known as mixed model assembly lines (MMALs). In MMALs, all models with the same standardised platform but different customisable product attributes are classified in the same family [1]. General-propose machines with automated tool changing equipment and highly flexible operators are necessary to realise an arbitrarily intermixed sequence of various models of a standardised product with similar process requirements to be assembled on the same line at negligible setup costs.

Typically, workstations on the assembly line are aligned straight along a conveyer belt. Monotone and boring types of work in the straight line layout may not challenge the working enthusiasm of operators, as well as being inflexible to manage changes in external environ- ments. As a consequence of just-in-time (JIT) implementation, manufacturers aim to achieve continuously improved productivity, cost, and product quality by eliminating all wastes in their production systems [2]. However, the straight line cannot fully support the adoption of JIT principles to manufacturing especially in the utilisation of multi-skilled operators. Hence, such companies as Allen-Bradley and GE have replaced their traditional straight lines with U-shaped production lines, called U-lines hereafter [3]. Figure 1 shows the configuration of the U-line.

In the U-line, the entrance and the exit are placed on the same position. A rather narrow U-shape is normally formed since both ends of the line are located closely

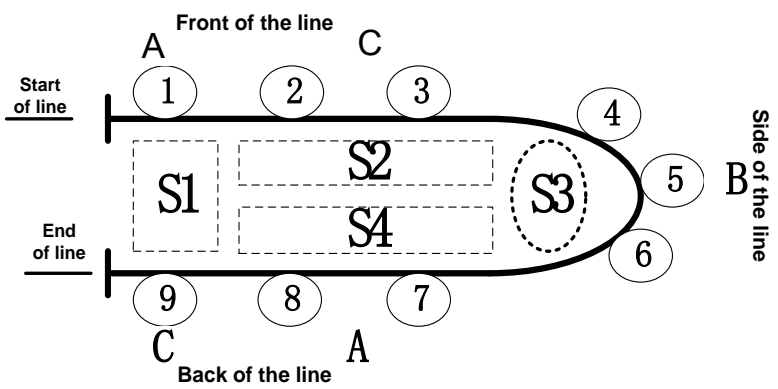

Figure 1. Configuration of a U-shaped assembly line 
together. Tasks are arranged around the U-line and are organised into workstations. A part of the U-line comprising a set of tasks with the same directional alignment as the entrance is called front zone. The opposite side of the front zone where the exit-side is located is called back zone. The set of tasks joining front and back zones and being the base of the U-line is located in side zone. Two kinds of workstations can be formed in the U-line. A regular workstation comprises tasks located sequentially along the front (S2), back (S4), or side (S3) of the U-line; whereas, a crossover workstation (S1) includes tasks located on both the front and back of the line.

Multi-skilled operators are located inside of the line. Since some certain models have to visit a crossover workstation twice, the operator in charge of that crossover workstation may have to process two different models in the same cycle. For example, operator S1 performs task 1 of model A on the front side of the line, travels to the back side of the line to perform task 9 of model $\mathrm{C}$, and then returns to the front side of the line to begin the next cycle. The salient characteristic of crossover workstations of the U-line poses additional challenges for improved performances.

Compared to the straight-line, the U-line gains popularity from its benefit offerings including improving visibility and communication, operator flexibility, rotatable multi-skilled operators, know-how sharing, enhancing teamwork, better quality control, prompt problem solving, faster corrective action on rework, higher product quality, easily adjustable output rate, volume flexibility, eliminating the need for special material handling equipment, fewer workstations, higher machine utilisation, and higher line effectiveness for breakdown prone systems [4-7].

The U-line is an inevitable element and becomes a cornerstone to obtain the main benefits of JIT production principles, i.e. one-piece flow manufacturing, smoothened workload, and multi-skilled workforce. The U-line is expected to gain much more popularity in industries in the future. The survey found that nearly $75 \%$ of available U-lines are configured to produce a product with different models or more than one type of product on the same line [8]. This type of production is called a mixed-model U-line (MUL). MUL has gradually superseded traditional mixed-model straight line due to its greater efficiency offerings, e.g. productivity, flexibility, cost, adaptability to demand changes, machine utilisation, and quality [9].

Successful utilisation of MUL needs effective solutions to mixed-model U-line balancing (MULB) and mixed-model U-line sequencing (MUS). MULB, a long to medium-term decision with a typical planning horizon of several months, is a problem of determining the number and sequence of workstations on the line or the cycle time of the line to accommodate the different models of products; whereas MUS, a short-term decision normally revising on a daily basis, is a problem of determining a production sequence of mixed models introduced to the line to achieve given objectives. Although these two problems are heavily interrelated, they are normally addressed independently and hierarchically due to their own computational complexities involved. This paper will focus on the MULB problem.

A great deal of research has been conducted on the line balancing problem since it was first published in mathematical form by Salveson [10]. Comprehensive literature reviews presented by [3,11-14]. Boysen et al. [15] indicated that very little has been done concerning the U-line balancing problem. Since Monden [4] brought U-lines to the attention of research community, the first pioneer study of the U-line balancing problems was published by Miltenburg and Wijngaard [5]. They developed a dynamic programming (DP) procedure for a single-model U-line to determine the optimal balance for Type I of U-line balancing problems (minimum number of workstations) with up to 11 tasks. However, DP was reported impractical for obtaining optimal balances for large-sized problems. They then developed a single-pass heuristic namely U-line maximum ranked positional weight to use for larger problems (111 tasks) where the priority of each task is given to either the time required to complete both that task and all the tasks that must succeed or must precede it, whichever is larger. The heuristics showed satisfactory performance for large-sized problems. Sparling and Miltenburg [16] proposed an approximate solution algorithm to solve the MMUALB problem up to 25 tasks. The algorithm transformed the multi-model problem into an equivalent single-model problem. The optimal balance was solved by branch and bound algorithm with exponential computational requirement to find minimum number of workstations. Smoothing algorithm was used to adjust the initial balance to reduce the level of model imbalance. Miltenburg [17] presented a reaching dynamic algorithm to balance and rebalance a U-line facility that consists of numerous U-lines connected by multiline stations. The objective when balancing such a facility is to assign tasks to a minimum number of stations while satisfying cycle time, precedence, location, and station-type constraints. A secondary objective is to concentrated idle time in a single station. The proposed algorithm can solve U-line balancing problem with no more than 22 tasks without wide, sparse precedence graphs.

Urban [18] presented an integer programming formulation for determining the optimal balance for the U-line balancing (ULB) problem. By eliminating some variables through the use of bounds, the size of the model was reduced. It was shown that the proposed formulation can optimally balance larger problems (21 to 45 tasks) than the DP procedure of Miltenburg and Wijngaard [5]. Ajenblit and Wainwritght [19] were the first who applied a genetic algorithm (GA) for Type I ULB problems with 
the objectives of minimising total idle time, balancing workload among stations, or a combination of both. Several algorithms for assigning tasks to workstations were proposed. The fitness value of a chromosome is determined by applying all these algorithms to it and the one with lowest fitness value is selected. They found that these assignment algorithms proved to have merit and GA proved to be computationally efficient. Scholl and Klein [20] considered different types of the ULB problem, i.e. Type I, Type II, and Type E. A new branch and bound procedure called ULINO (U-line optimiser) adapted from their previous algorithm developed for the straight-shaped problem called SALOME was proposed. Computational results of up to 297-task problems showed that the procedures yielded promising results in limited computation time.

Erel et al. [21] proposed an algorithm with a coupling of a solution generator module and a simulated annealing (SA) module. The generator assigned tasks sequentially to separate stations and combines two adjacent stations with minimum total station time until infeasibility is found. Then, SA reconstructed feasibility for such solutions by reassigning the tasks in the combined station to other stations by minimizing the maximum station time. The algorithm was tested on a variety of data sets with up to 297 tasks and found quite effective. Aase et al. [22] proposed a branch-and-bound (B\&B) solution procedure called U-OPT (U-line OPTimisation) for a ULB problem. Four design elements of the $B \& B$ procedure are investigated including branching strategies, fathoming criteria, heuristics to obtain upper bounds at each node, and identification of initial setting solutions. Paired-task lower bound was largely responsible for the dominance in the efficacy of U-OPT over existing methods. Aase et al. [23] conducted empirical experiments to confirm that the U-shaped layout can significantly improve labour productivity over the traditional straight-line one. Interestingly, the improvement tends to be higher during high demand periods when operators are assigned three or fewer tasks on average, when the problem size is small, and when assembly sequence is fairly well structured.

Martinez and Duff [24] applied heuristic rules adapted from the simple line balancing problem to the Type I UALB problems up to 21 tasks. Some heuristics were found to produce optimal results. To achieve improved solutions, each gene in a chromosome of GA representing the heuristic rule was used to break ties during the task assignment process. Balakrishnan et al. [25] modified 13 single-pass heuristics to balance U-lines with the existent of travelling time and investigated their effectiveness under various problem conditions. Gokcen and Agpak [26] developed a goal programming model for the ULB problems up to 30 tasks. This approach offers increased flexibility to the decision maker since conflicting goals can be dealt with at the same time. Urban and
Chiang [27] considered the ULB problem with stochastic task times and developed a linear, integer program using a piecewise approximation for the chance constraints to find the optimal solution. The proposed method effectively solved practical-sized problems optimally up to 28 tasks. Chiang and Urban [28] developed a hybrid heuristics comprising an initial feasible solution module and a solution improvement module for the stochastic ULB problem. The heuristic can identify optimal or near-optimal solutions for up to 111-task problems. Kara et al. [29] developed a binary fuzzy goal programming for 8-task ULB with fuzzy goals that allow decision makers to consider the number of workstations and cycle time as imprecise values.

Baykasoglu [30] proposed multi-objective SA for ULB problems with the aim of maximising smoothness index and minimising the number of workstations. Task assignment rules were used in constructing feasible solutions. The optimal solutions for each problem were found in short computation times. Hwang et al. [31] developed a priority-based genetic algorithm (PGA) for ULB problems for up to 111 tasks. A weighted-sum objective function comprising the number of workstations and the workload variation was considered. The proposed model obtained improved workload variation, especially for large size problems. Hwang and Katayama [32] proposed an extension version of PGA namely an amelioration structure with genetic algorithm (ASGA) to deal with workload balancing problems in mixed-model U-shaped lines for up to 111 tasks. ASGA was able to find better solutions than PGA in terms of workload variation. Boysen and Fliedner [33] proposed a general solution procedure for U-shaped assembly line balancing using an ant colony optimisation (ACO) approach. Their procedure was versatile in the sense that various line balancing features found in practice can be incorporated into the model. Baykasoglu and Dereli [33] proposed ACO that integrates COMSOAL and ranked positional weight heuristics for solving ULB problems. The proposed algorithm found optimum solutions in short computational times.

The existent of crossover workstations in MUL opens a chance for MUS, apart from MULB, to smoothen workload distribution among workstations since the crossover workstation allows a model mix to be processed in a cycle. As a result, MULB and MUS can play significant roles in workload smoothening of MUL. Since these problems are highly correlated, especially when the workload smoothening objective needs to be achieved, several researchers have attempted to solve these two problems simultaneously in an aggregated manner. Miltenburg [34] modelled the joint problems of line balancing and model-sequencing for mixed-model U-lines operated under a JIT environment and proposed a solution algorithm for solving both problems simultane- 
ously. Kim et al. [35] developed a symbiotic evolutionary algorithm called co-evolutionary algorithm (PCoA), which imitates the biological co-evolution process through symbiotic interaction, to handle the integration of balancing and sequencing problem in MUL for up to 111 tasks. Later, Kim et al. [36] proposed an endosymbiotic evolutionary algorithm (EEA), an extended version of the symbiotic evolutionary algorithm, to simultaneously solve line balancing and sequencing in MUL. The proposed algorithm obtained much better quality solutions than PCoA and a traditional hierarchical approach. Agrawal and Tiwari [37] demonstrated the superiority of the collaborative ant colony optimisation in simultaneously tackling disassembly line balancing and sequencing problem in MUL for up to 80 tasks. Sabuncuoglu et al. [38] developed a family of ant colony algorithms that make both sequencing and task assignment decisions simultaneously for ULB problems up to 111 tasks.

Kara et al. [9] proposed SA to deal with a multi-objective approach for balancing and sequencing MULs in JIT production systems for up to 30 tasks to simultaneously minimise the weighted sum of the absolute deviations of workloads across workstations, part usage rate, and cost of setups. Kara et al. [39] proposed SA based heuristic approach for solving balancing and sequencing problems of mixed-model U-lines simultaneously for up to 30 tasks. SA was capable of minimising the number of workstations and minimising the absolute deviation of the workloads among workstations. Kara [40] presented a mixed, zero-one integer, nonlinear programming for mixed-model U-line balancing and sequencing problems for up to 111 tasks with the objective of minimising absolute deviation of workloads. An efficient SA was also proposed and its performance outperformed PCoA and EEA.

Literature has demonstrated that the MULB is an important problem for modern assembly systems operated under JIT environment. Although several exact methods for their solutions were proposed, only small sized problems can be optimally solved due to the complexity of the problem. Hence, a computational more effective algorithm is needed for larger sized problem. Also, the algorithm has to be able to easily handle multiple objectives simultaneously. In this paper, such an algorithm that utilises the concept of evolutionary algorithm namely combinatorial optimization with coincidence algorithm (COIN) is proposed for multi-objective MULB problems. Three objectives including minimum number workstations, minimum work relatedness, and minimum workload smoothness are considered simultaneously. The performances of COIN are compared with a well-known algorithm namely non-dominated sorting genetic algorithm II (NSGA II) and their memetic versions The purpose of this study is to see the feasibility and effectiveness of the COIN approach which is one of the most recent meta-heuristics to solve this well-known problem and compare it against others in terms of quality of solutions and solution time.

The organisation of this paper is as follows. In the next section, the detailed description of the multi-objective optimisation problem is presented, followed by an explanation of the multi-objective MULB problems. The proposed algorithm to solve MULB problems is elaborated next, and the experimental design and results are explained respectively. Finally, the concluding remark of the research is given.

\section{Multi-Objective Evolutionary Algorithms}

A multi-objective optimisation problem (MOP) is related to the problem where two or more objectives have to be optimised simultaneously. Generally, such objectives are conflicting and represented in different measurement units, preventing simultaneous optimisations of each one. MOP can be formulated, without loss of generality, as follows:

$$
\text { Minimize }_{x \in \Omega}\left\{f_{1}(x), f_{2}(x), \ldots, f_{k}(x)\right\}
$$

where solution $x$ is a vector of decision variables for the considered problem; $\Omega$ is the feasible solution space; and $f_{i}(x)$ is the $i^{\text {th }}$ objective function $(i=1,2, \ldots, k)$. Two approaches often employ to solve MOP. The first approach is to combine each objective function into a single composite function, e.g. weighted sum method, utility theory, etc. The advantage of this method is straightforward computation. However, two practical problems are often experienced with this approach: 1) selection of the suitable weights can be very difficult even for those who are unfamiliar with the problem and 2) small perturbations in the weights can sometimes lead to totally different solutions [41]. As a result, the second approach, e.g. multi-objective evolutionary algorithms (MOEAs), has come into play. This approach determines a set of alternative solutions for (1) rather than a single optimal solution. These solutions are optimal in the wider sense such that no other solutions in the search space are superior to them when all objectives are considered. A decision vector $x$ is said to dominate a decision vector $y$ (also written as $x>y$ ) if:

$$
f_{i}(x) \leq f_{i}(y) \text {, for all } i \in\{1,2, \ldots, k\}
$$

and $f_{i}(x)<f_{i}(y)$, for at least one $i \in\{1,2, \ldots, k\}$

All solutions that dominate the others but do not dominate themselves are called non-dominated (superior) solutions. A Pareto-optimal solution is a global optimal solution which is not dominated by any other solutions in the feasible solution space. A set that contains all feasible Pareto-optimal solutions is called a Pareto-optimal set or efficient set. The collection of the points of the Pareto-optimal set (or the corresponding images of the Pareto-optimal set) along a curve in the 
objective space that has a set of attributes collectively dominating all other points not on the frontier are termed the Pareto-optimal frontier (front) or efficient frontier (front). An example of the Pareto-optimal solutions for a two-objective minimisation problem is illustrated in Figure 2. It is obvious that an amount of sacrifice in one objective is always incurred to achieve a certain amount of gain in the other (inverse relationship) while moving from one Pareto-optimal solution to another. Providing Pareto-optimal solutions to the decision maker is more preferable to a single solution since practically, when considering real-life problems, a final decision is always based on a trade-off between conflicting objective functions.

MOEAs have recently become popular and have been applied to a wide range of problems from social to engineering problems [42]. In general, MOEAs are ideally suited to MOP because they are capable of searching a whole set of multiple Pareto-optimal solutions in a single run. In addition, the shape or continuity of the Pareto-optimal frontier has less effect to MOEAs than traditional mathematical programming. The approximation of a true Pareto-optimal set involves two conflicting objectives: 1.) the distance to the true Pareto frontier is to be minimised, and 2.) the diversity of the evolved solutions is to be maximised [43]. To achieve the first objective, a Pareto-based fitness assignment is normally designed to guide the search toward the true Pareto-optimal frontier [44, 45].

In the view of the second objective, some MOEAs successfully provide density estimation methods to preserve the population diversity. Although several versions of MOEAs have been developed [42], non-dominated sorting genetic algorithms-II (NSGA II) [46] is among the most promising one in terms of convergence speed to Pareto-optimal solutions and even distribution of the

Minimize $f_{2}(x)$

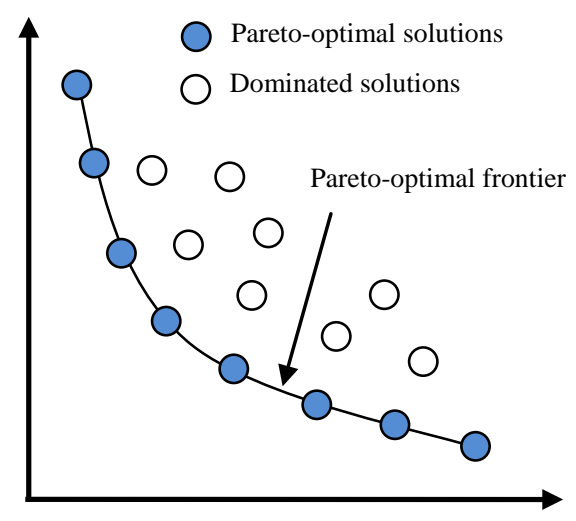

Minimize $f_{1}(x)$

Figure 2. Pareto-optimal solutions
Pareto frontier. NSGA II is an elitist multi-objective genetic algorithm being introduced by Deb et al. (2002). It uses a fixed population size of $N$ for both parent and offspring populations. Once a new offspring population is created, it is combined with its parent population. The size of the combined population becomes $2 N$. A non-dominated sorting method is used to identify Pareto frontiers $\left(F_{1}, F_{2}, \ldots, F_{k}\right)$ in the combined population. The first frontier $\left(F_{1}\right)$ is the best in the combined population. The next population (archive) is created by selecting frontiers based on their rankings; the best Pareto frontier being selected first. If the number of members in the archive is smaller than the population size $(N)$, the next frontier will be selected and so on. If adding a frontier would increase the number of members in the archive to exceed the population size, a truncation operator is applied to that frontier based on the crowded tournament selection by which the winner of two same rank solutions is the one that possesses the greater crowding distance (farther apart from its neighbours). This is to maintain a good spread of solutions in the obtained set of solutions.

Memetic algorithms (MAs), a type of evolutionary algorithms (EAs), have been recognised as a powerful algorithmic paradigm on complex search spaces for evolutionary computing [47]. MAs are inspired by models of adaptation in nature systems that combine evolutionary adaptation of populations of individuals with individual learning with a lifetime. A meme is a unit of information that reproduces itself while people exchange ideas. Memes are adapted by the people who transmit them before being passed on to the next generation. MAs use EAs to perform exploration and use local search to exercise exploitation. A separate local search algorithm can be applied to improve the fitness of individuals by special hill-climbing. Local search in MAs is similar to simple hill-climbing with differences in that 1) the neighbourhood of the current solutions is searched systematically instead of random searching in the space of all candidate solutions, and 2) the neighborhood search is repeated until a locally optimal solution is found. An advantage of local search in MAs over other heuristics is that local exploitation around individual can be performed much more effectively; hence, good solutions in a small region of the search space can be found quickly.

\section{Multi-Objective MULB Problem}

\subsection{MULB Problem}

To plan an assembly process for any product on an assembly line, its total amount of work is partitioned into a set of elementary operations namely tasks. Assembly line balancing is the allocation of a set of tasks to workstations without violating any constraints to optimize some measure of performance. Typical constraints include 
each task is allocated to one and only one workstation, precedence relationship that reflects technological and organisational constraints among the tasks is not violated, and total task time of any workstation does not exceed the given cycle time [13].

To perform a task on a workstation, not only tools, equipment, machinery, and labour skills have to be selected properly, but also its precedence relationship has to be followed strictly. A precedence diagram is often used to visually demonstrate such relationship. Nodes, node weights, and arrows on the precedence diagram represent tasks, task times, and precedence constraints between tasks, respectively. For MMAL, a merged precedence diagram is needed which can be created as follows [16].

1) Compute the weighted average task time for each task. Let $\mathrm{M}=$ the number of models to be produced during a planning horizon, $D_{m}=$ the demand of product model $\mathrm{m}(\mathrm{m}=1,2, \ldots, \mathrm{M})$ task i $(\mathrm{i}=1,2, \ldots, \mathrm{N})$ of model $M$ has task time $=t_{i m}$, The weighted average task time $t_{i}$ is

$$
t_{i}=\sum_{m=1}^{M}\left\{D_{m} t_{i m}\right\} / \sum_{m=1}^{M} D_{m}
$$

2) Merge the precedence diagram of each model to form the merged precedence diagram. It is assumed that the precedence relationship is consistent from model to model. The merged precedence diagram (MPD) is created by adding arrow $x y$ to MPD if, for any model, task $x$ is an immediate predecessor of task $y$.

MULB is more complex than the traditional straight line since not only can the set of assignable tasks be considered from the set of tasks whose predecessors have already been assigned (moving forward through MPD and allocating tasks on the front side of the U-line) as the straight line, but also from the set of tasks whose successors have already been assigned (moving backwards through allocating tasks on the back side of the U-line). This permission increases possibility on how to allocate tasks to workstations and often leads to a fewer number of workstations than the straight line. Based on MPD, literature always assumes that each task type is assigned to one and only one workstation regardless of the model [32].

\subsection{Objective Functions}

Although several measures can be used to evaluate the performance of line balancing in MUL, in this paper three main objectives that support JIT implementation to be simultaneously optimised are evaluated including number of workstations, variation of workload, and variation of work relatedness. Since the type I problem of MULB is considered, a fixed cycle time, assembly task time, and precedence relationship are given and our first objective is to minimize the number of workstations. Achieving this objective can result in low labour cost and less space requirement. If $m$ is the number of work- station, the objective function is formulated as follows.

$$
f_{1}(x)=\text { minimize } m
$$

The second objective is to smooth (minimize variation of) the workloads distributed across workstations. Several benefits can be gained when MUL is operated in this manner including increased production rate, reduced line congestion, but more importantly, mitigates the concerns of inequity in task assignments among workers [35]. The workload smoothness objective can be formulated as follows.

$$
f_{2}(x)=\operatorname{minimize} \sqrt{\sum_{k=1}^{m}\left(S_{\max }-S_{k}\right)^{2} / m}
$$

where $S_{k}=$ total time of workstation $k, S_{\max }=$ maximum total time of all workstations $=\operatorname{maximize}_{k}(k=$ $1,2, \ldots, m)$.

The third objective is to minimize variation of work relatedness in a workstation. The purpose of this objective is to allocate interrelated tasks to the same workstation as many as possible. Not only can such an assignment improve work efficiency, but it is also useful to assembly line designers since they may have greater flexibility in locating facilities and workstations. The formulation of this objective is as follows.

$$
f_{3}(x)=\operatorname{minimize}\left\{m-m / \sum_{k=1}^{m} S N_{k}\right\}
$$

where $S N_{k}=$ number of relatedness of tasks in workstation $k$.

Although three objectives are considered simultaneously in this paper, for type I problem, the first objective dominates the others. As a result, if there are two candidate solutions, the one with lower number of workstations will always selected regardless of how good the other two objectives are.

\section{Proposed Algorithm}

\subsection{COIN}

Wattanapornprom et al. [48] developed a new effective evolutionary algorithm called combinatorial optimisation with coincidence (COIN) originally aiming for solving travelling salesman problems. The idea is that most well-known algorithms such as GA search for good solutions by sampling through crossover and mutation operations without much exploitation of the internal structure of good solution strings. This may not only generate large number of inefficient solutions dissipated over the solution space but also consuming long CPU time. In contrast, COIN considers the internal structure of good solution strings and memorises paths that could lead to good solutions. COIN replaces crossover and mutation operations of GA and employs joint probability matrix as a means to generate solutions. It prioritises the selection of the paths with higher chances of moving towards good solutions.

Apart from traditional learning from good solutions, 
COIN allows learning from below average solutions as well. Any coincidence found in a situation can be statistically described whether the situation is good or bad. Most traditional algorithms always discard the bad solutions without utilising any information associated with them. In contrast, COIN learns from the coincidence found in the bad solutions and uses this information to avoid such situations to recurrent; meanwhile, experiences from good coincidences are also used to construct better solutions (Figure 3). Consequently, the chances that the paths always being parts of the bad solutions are used in the new generations are lessened. This lowers the number of solutions to be considered and hence increases the convergence speed.

COIN uses a join probability matrix (generator) to create the population. The generator is initialised so that it can generate a random tree with equal probability for any configuration. The population is evaluated in the same way as traditional EAs. However, COIN uses both good and bad solutions to update the generator. Initially, COIN searches from a fully connected tree and then incrementally strengthening or weakening the connections. As generations pass by, the probabilities of selection certain paths are increased or decreased depending on the incidences found in the good or bad solutions. The algorithm of COIN can be stated as follows.

1) Initialise the joint probability matrix (generator).

2) Generate the population using the generator.

3) Evaluate the population.

4) Diversity preservation.

5) Select the candidates according to two options: (a) good solution selection (select the solutions in the first rank of the current Pareto frontier), and (b) bad solution selection (select the solutions in the last rank of the current Pareto frontier).

6) For each joint probability matrix $H\left(x_{i} / x_{j}\right)$, adjust the generator according to the reward and punishment scheme as (4).

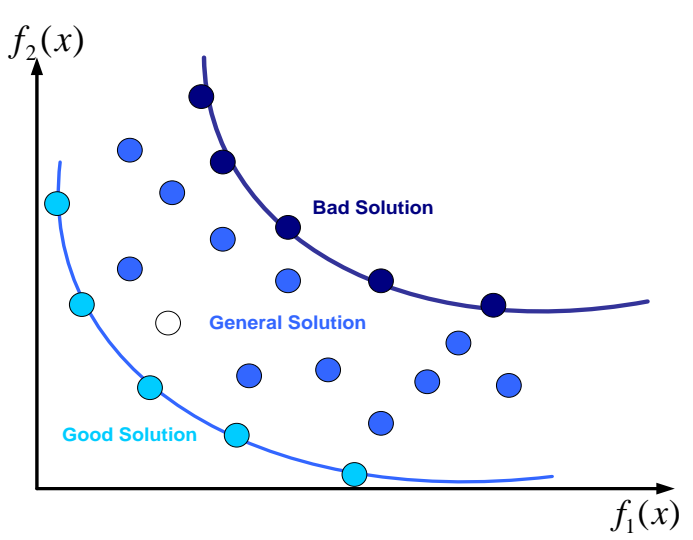

Figure 3. Good and bad solutions

$$
\begin{aligned}
& x_{i, j}(t+1)=x_{i, j}(t)+\frac{k}{\left(n-1-n p_{i}\right)}\left\{r_{i, j}(t+1)-p_{i, j}(t+1)\right\} \\
& \quad+\frac{k}{\left(n-1-n p_{i}\right)^{2}}\left\{\sum_{j=1}^{n} p_{i, j}(t+1)-\sum_{j=1}^{n} r_{i, j}(t+1)\right\}
\end{aligned}
$$

where $x_{i, j}=$ the element $(i, j)$ of joint probability matrix $H\left(x_{i} / x_{j}\right), k=$ the learning coefficient, $r_{i, j}=$ the number of coincidences $\left(x_{i}, x_{j}\right)$ found in the good solutions, $p_{i, j}=$ the number of coincidences $\left(x_{i}, x_{j}\right)$ found in the bad solutions, $t=$ generation number, $n=$ the size of the problem, and $n p_{i}=$ number of the direct predecessors of task $i$.

7) Apply a strategy to maintain elitist solutions in the population, and then repeat Step 2 until the terminating condition is met.

\subsection{Numerical Example}

The 11-task problem originated by Jackson [49] and later extended to accommodate a product mix by Hwang and Katayama [31] is used to elaborate the algorithm of COIN. Three models (A, B, and $\mathrm{C}$ ) of the product mix with an equal minimum part set $(\mathrm{MPS}=[1,1,1])$ are produced on MUL with 10-minute cycle time. Their precedence diagrams are shown in Figure 4.

\section{Joint Probability Matrix Initialization}

The number of tasks to be considered is 11 . Therefore, the dimension of from-to joint probability matrix $H\left(x_{i} / x_{j}\right)$ is $(11 \times 11)$. The value of each element $\left(x_{i, j}\right)$ in the matrix is the probability of selecting product $j$ after product $i$. In order to incorporate some precedence relationship into the matrix, in each row, the element which belongs to the direct predecessor of the task is set to 0 to prohibit producing such task before its direct predecessor. For example, the direct predecessor of task 2 is task 1 ; hence, $x_{2,1}=0$. Also, $x_{2,2}=0$, since it cannot move within itself. Initially, the value of the remaining elements in the $2^{\text {nd }}$ row of the matrix is equal to $1 /\left(n-1-n p_{2}\right)$ $=1 /(11-1-1)=0.111$. Continue this computation for all the remaining tasks (rows), the initial joint probability matrix is shown in Table $\mathbf{1 .}$

\section{Population Generation}

The order representation scheme is used to create chromosomes. The task order list in a chromosome is created by moving forward through MPD. If there is more than one task can be selected, the probability of selecting any task will depend on its value on the join probability matrix. For example, task 1 is selected for the first position since it is the only task to be considered. After selecting task 1 , the set of eligible tasks comprises tasks 2, 3, 4 and 5. From row 1 of the joint probability matrix, a job is randomly selected according to its probability of selection $\left(p_{1, i}=0.1000\right.$, for $\left.i=2, \ldots, 11\right)$. If the selected job is not in the set of eligible tasks, redo the 


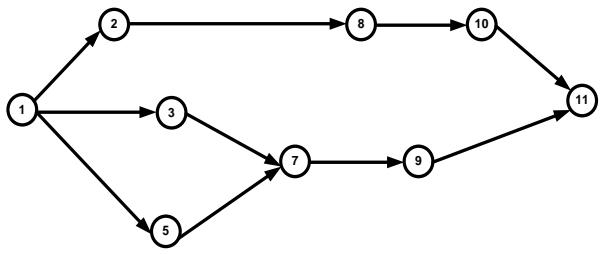

(a)

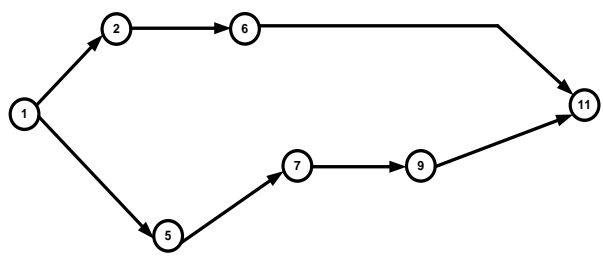

(c)

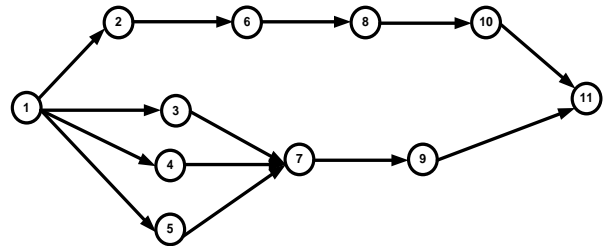

(b)

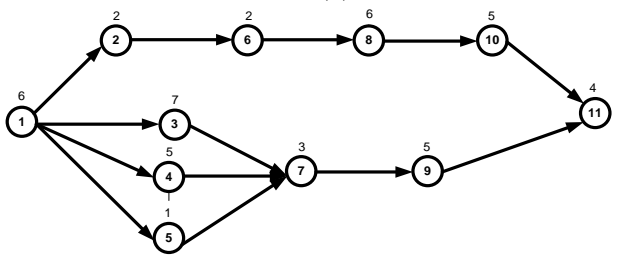

(d)

Figure 4. Precedence diagrams. (a) Precedence diagram of model A; (b) Precedence diagram of model B; (c) Precedence diagram of model C; (d) Merged precedence diagram of models A, B and C

Table 1. Initial joint probability matrix

\begin{tabular}{|c|c|c|c|c|c|c|c|c|c|c|c|}
\hline $\bar{j}$ & 1 & 2 & 3 & 4 & 5 & 6 & 7 & 8 & 9 & 10 & 11 \\
\hline 1 & 0 & 0.1000 & 0.1000 & 0.1000 & 0.1000 & 0.1000 & 0.1000 & 0.1000 & 0.1000 & 0.1000 & 0.1000 \\
\hline 2 & 0 & 0 & 0.1111 & 0.1111 & 0.1111 & 0.1111 & 0.1111 & 0.1111 & 0.1111 & 0.1111 & 0.1111 \\
\hline 3 & 0 & 0.1111 & 0 & 0.1111 & 0.1111 & 0.1111 & 0.1111 & 0.1111 & 0.1111 & 0.1111 & 0.1111 \\
\hline 4 & 0 & 0.1111 & 0.1111 & 0 & 0.1111 & 0.1111 & 0.1111 & 0.1111 & 0.1111 & 0.1111 & 0.1111 \\
\hline 5 & 0 & 0.1111 & 0.1111 & 0.1111 & 0 & 0.1111 & 0.1111 & 0.1111 & 0.1111 & 0.1111 & 0.1111 \\
\hline 6 & 0.1111 & 0 & 0.1111 & 0.1111 & 0.1111 & 0 & 0.1111 & 0.1111 & 0.1111 & 0.1111 & 0.1111 \\
\hline 7 & 0.1429 & 0.1429 & 0 & 0 & 0 & 0.1429 & 0 & 0.1429 & 0.1429 & 0.1429 & 0.1429 \\
\hline 8 & 0.1111 & 0.1111 & 0.1111 & 0.1111 & 0.1111 & 0 & 0.1111 & 0 & 0.1111 & 0.1111 & 0.1111 \\
\hline 9 & 0.1111 & 0.1111 & 0.1111 & 0.1111 & 0.1111 & 0.1111 & 0 & 0.1111 & 0 & 0.1111 & 0.1111 \\
\hline 10 & 0.1111 & 0.1111 & 0.1111 & 0.1111 & 0.1111 & 0.1111 & 0.1111 & 0 & 0.1111 & 0 & 0.1111 \\
\hline 11 & 0.1250 & 0.1250 & 0.1250 & 0.1250 & 0.1250 & 0.1250 & 0.1250 & 0.1250 & 0 & 0 & 0 \\
\hline
\end{tabular}

selection. Suppose we select task 5 , the new set of eligible tasks becomes tasks 2, 3 and 4 . Continue this mechanism until all positions in the task order list are filled and we obtain the task order list of $L_{1}=\{1,5,3,4,7,2,6,9$, $8,10,11\}$. Assume that the population size is 5 and the four remaining initial population consists of chromosomes $L_{2}=\{1,4,5,3,7,9,2,6,8,10,11\} ; L_{3}=\{1,3,2,6,8,5,10$, $4,7,9,11\} ; L_{4}=\{1,4,3,2,6,8,10,5,7,9,11\}$, and $L_{5}=\{1,5,4$, 3,2,6,8,7,9,10,11\}.

\section{Population Evaluation}

To find tentative tasks to be allocated on the U-line, we have to search through the task order list in both forward and backward directions. The tentative task on forward or backward searching is the first found task that has its task time less than or equal to the remaining workstation cycle time and does not violated MPR. If both forward and backward tentative tasks are found, either one is selected randomly. But if none is found and the task order list still has some task not yet being allocated, a new workstation is opened. For example, for the task or- der list of $L_{1}=\{1,5,3,4,7,2,6,9,8,10,11\}$ and cycle time $c$ $=10$, the forward and backward tentative tasks are tasks 1 and 11 . If task 1 is randomly selected, the remaining cycle time is $10-6=4$, the new forward and backward tentative tasks are tasks 5 and 11 so on and so forth. Finally, a feasible line balance with $m=7$ workstations and workstation load distribution given by $S_{1}=\{1,5\}, S_{2}=\{10,11\}$, $S_{3}=\{8\}, S_{4}=\{9\}, S_{5}=\{3\}, S_{6}=\{4,6,7\}, S_{7}=\{2\}$. Repeat this procedure for the remaining task order lists to obtain the number of workstations and workstation load distribution for each of them. Having obtained feasible line balances, three objectives have to be evaluated for each chromosome. Table 2 indicates that all chromosomes give the same number of workstations; therefore, they are all eligible for Pareto ranking based on workload smoothness and work relatedness objectives. The Pareto ranking technique proposed by Goldberg [50] is used to classify the population into non-dominated frontiers and a dummy fitness value (lower value is better) is assigned to each chromosome (Figure 5). 


\section{Diversity Preservation}

COIN employs a crowding distance approach [46] to generate a diversified population with uniformly spread over the Pareto frontier and avoid a genetic drift phenomenon (a few clusters of populations being formed in the solution space). The salient characteristic of this approach is that there is no need to define any parameter in calculating a measure of population density around a solution. The crowding distances computed for all solutions are infinite since only one solution is found for each frontier.

\section{Solution Selection}

Having defined the Pareto frontier, the good solutions are the chromosomes located on the first Pareto frontier (dummy fitness $=1$ ), i.e. $L_{2}=\{1,4,5,3,7,9,2,6,8,10,11\}$. The bad solutions are those located on the last Pareto frontier (dummy fitness = 5), i.e. $L_{4}=\{1,4,3,2,6,8,10,5,7,9,11\}$.

\section{Joint Probability Matrix Adjustment}

The adjustment of joint probability matrix is crucial to the performance of COIN. Reward will be given to $x_{i, j}$ if the order pair $(i, j)$ is in the good solution to increase the chance of selection in the next round. For example, an order pair $(1,4)$ is in the good solution $L_{2}=\{1,4,5,3,7,9,2,6$, $8,10,11\}$. Assume that $k=0.3$, hence the value of $x_{i, j}$ where $i=1$ and $j=4$ is increased by $k /\left(n-1-n p_{1}\right)$ $=0.3 /(11-1-0)=0.03$. The updated value of $x_{i, j}$ of the order pair $(1,4)$ becomes $0.1+0.03=0.13$. The values of the other order pairs located in the same row of the order pair $(1,4)$ is reduced by $k /\left(n-1-n p_{1}\right)^{2}=0.3 / 100=$ 0.003 . For example, the value $x_{i, j}$ where $i=1$ and $j=4$ is $0.1-0.003=0.0970$. Continue this procedure to all order pairs located in the good solution; the revised joint probability matrix is obtained (Table 3 ).

In contrast, if the order pair $(i, j)$ is in the bad solution, $x_{i, j}$ will be penalised to reduce the chance of selection in the next round. For example, an order pair $(1,4)$ is in the bad solution $L_{4}=\{1,4,3,2,6,8,10,5,7,9,11\}$. Therefore, the value of $x_{i, j}$ where $i=1$ and $j=4$ is decreased by $k /\left(n-1-n p_{1}\right)=0.3 / 10=0.03$. The updated value of $x_{i, j}$ of the order pair $(1,4)$ becomes $0.130-0.030=0.100$. The values of the other order pairs located in the same row of the order pair $(1,4)$ is increased by $k /\left(n-1-n p_{1}\right)^{2}$ $=0.3 / 100=0.003$. For example, the value $x_{i, j}$ where $i=$ 1 and $j=2$ is $0.097+0.003=0.100$. Continue this pro- cedure to all order pairs located in the bad solution; the revised joint probability matrix is obtained (Table 4).

\section{Elitism}

To keep the best solutions found so far to be survived in the next generation, COIN uses an external list with the same size as the population size to store elitist solutions. All non-dominated solutions created in the current population are combined with the current elitist solutions. Goldberg's Pareto ranking technique is used to classify the combined population into several non-dominated frontiers. Only the solutions in the first non-dominated frontier are filled in the new elitist list. If the number of solutions in the first non-dominated frontier is less than or equal to the size of the elitist list, the new elitist list will contain all solutions of the first non-dominated frontier. Otherwise, Pareto domination tournament selection [51] is exercised. Two solutions from the first non-dominated solutions are randomly selected and then the solution with larger crowding distance measure and not being selected before is added to the new elitist list. This approach not only ensures that all solutions in the elitist list are non-dominated solutions but also promoting diversity of the solutions. According to our example, the current elitist list is empty and the solutions in the current first non-dominated frontier is $L_{2}=\{1,4,5,3,7,9,2,6,8,10$, $11\}$. When both sets are combined the non-dominated frontier is still the same. Also, the number of the combined solutions is less than the size of the elitist list; hence, both solutions are added to the new elitist.

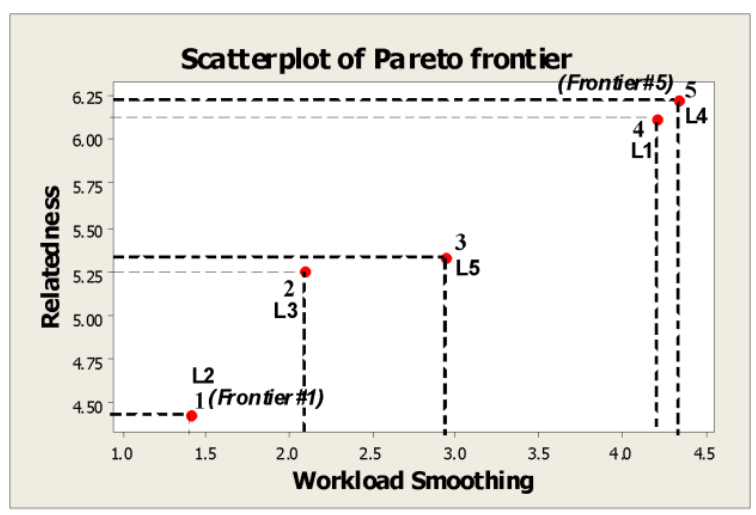

Figure 5. Pareto frontier of each chromosome

Table 2. Objective functions of each chromosome

\begin{tabular}{cccccc}
\hline $\begin{array}{c}\text { Chromosome } \\
\text { Number }\end{array}$ & $\begin{array}{c}\text { Number of } \\
\text { Workstations }\end{array}$ & $\begin{array}{c}\text { Workload } \\
\text { Smoothness }\end{array}$ & $\begin{array}{c}\text { Work } \\
\text { Relatedness }\end{array}$ & Pareto Frontier & $\begin{array}{c}\text { Crowding } \\
\text { Distance }\end{array}$ \\
\hline 2 & 4 & 1.4142 & 4.4444 & 1 & Infinite \\
3 & 4 & 2.0817 & 5.2500 & 2 & Infinite \\
5 & 4 & 2.9439 & 5.3333 & 3 & Infinite \\
1 & 4 & 4.2088 & 6.1250 & 4 & Infinite \\
4 & 4 & 4.3425 & 6.2222 & 5 & Infinite \\
\hline
\end{tabular}


Table 3. Revised joint probability matrix (good solution)

\begin{tabular}{|c|c|c|c|c|c|c|c|c|c|c|c|}
\hline $\mathrm{j}$ & 1 & 2 & 3 & 4 & 5 & 6 & 7 & 8 & 9 & 10 & 11 \\
\hline 1 & 0 & 0.0970 & 0.0970 & 0.1300 & 0.0970 & 0.0970 & 0.0970 & 0.0970 & 0.0970 & 0.0970 & 0.0970 \\
\hline 2 & 0 & 0 & 0.1074 & 0.1074 & 0.1074 & 0.1444 & 0.1074 & 0.1074 & 0.1074 & 0.1074 & 0.1074 \\
\hline 3 & 0 & 0.1074 & 0 & 0.1074 & 0.1074 & 0.1074 & 0.1444 & 0.1074 & 0.1074 & 0.1074 & 0.1074 \\
\hline 4 & 0 & 0.1074 & 0.1074 & 0 & 0.1444 & 0.1074 & 0.1074 & 0.1074 & 0.1074 & 0.1074 & 0.1074 \\
\hline 5 & 0 & 0.1074 & 0.1444 & 0.1074 & 0 & 0.1074 & 0.1074 & 0.1074 & 0.1074 & 0.1074 & 0.1074 \\
\hline 6 & 0.1074 & 0 & 0.1074 & 0.1074 & 0.1074 & 0 & 0.1074 & 0.1444 & 0.1074 & 0.1074 & 0.1074 \\
\hline 7 & 0.1367 & 0.1367 & 0 & 0 & 0 & 0.1367 & 0 & 0.1367 & 0.1858 & 0.1367 & 0.1367 \\
\hline 8 & 0.1074 & 0.1074 & 0.1074 & 0.1074 & 0.1074 & 0 & 0.1074 & 0 & 0.1074 & 0.1444 & 0.1074 \\
\hline 9 & 0.1074 & 0.1444 & 0.1074 & 0.1074 & 0.1074 & 0.1074 & 0 & 0.1074 & 0 & 0.1074 & 0.1074 \\
\hline 10 & 0.1074 & 0.1074 & 0.1074 & 0.1074 & 0.1074 & 0.1074 & 0.1074 & 0 & 0.1074 & 0 & 0.1444 \\
\hline 11 & 0.1250 & 0.1250 & 0.1250 & 0.1250 & 0.1250 & 0.1250 & 0.1250 & 0.1250 & 0 & 0 & 0 \\
\hline
\end{tabular}

Table 4. Revised joint probability matrix (bad solution)

\begin{tabular}{ccccccccccccc}
\hline $\mathrm{j}$ & 1 & 2 & 3 & 4 & 5 & 6 & 7 & 8 & 9 & 10 & 11 \\
$\mathrm{i}$ & & & & & & & & & & & \\
& & 0 & 0.1000 & 0.1000 & $\mathbf{0 . 1 0 0 0}$ & 0.1000 & 0.1000 & 0.1000 & 0.1000 & 0.1000 & 0.1000 & 0.1000 \\
2 & 0 & 0 & 0.1111 & 0.1111 & 0.1111 & $\mathbf{0 . 1 1 1 1}$ & 0.1111 & 0.1111 & 0.1111 & 0.1111 & 0.1111 \\
3 & 0 & $\mathbf{0 . 0 7 4 1}$ & 0 & 0.1111 & 0.1111 & 0.1111 & 0.1481 & 0.1111 & 0.1111 & 0.1111 & 0.1111 \\
4 & 0 & 0.1111 & $\mathbf{0 . 0 7 4 1}$ & 0 & 0.1481 & 0.1111 & 0.1111 & 0.1111 & 0.1111 & 0.1111 & 0.1111 \\
5 & 0 & 0.1111 & 0.1444 & 0.1111 & 0 & 0.1111 & $\mathbf{0 . 0 7 7 8}$ & 0.1111 & 0.1111 & 0.1111 & 0.1111 \\
6 & 0.1111 & 0 & 0.1111 & 0.1111 & 0.1111 & 0 & 0.1111 & $\mathbf{0 . 1 1 1 1}$ & 0.1111 & 0.1111 & 0.1111 \\
7 & 0.1429 & 0.1429 & 0 & 0 & 0 & 0.1429 & 0 & 0.1429 & $\mathbf{0 . 1 4 2 9}$ & 0.1429 & 0.1429 \\
8 & 0.1111 & 0.1111 & 0.1111 & 0.1111 & 0.1111 & 0 & 0.1111 & 0 & 0.1111 & $\mathbf{0 . 1 1 1 1}$ & 0.1111 \\
9 & 0.1111 & 0.1481 & 0.1111 & 0.1111 & 0.1111 & 0.1111 & 0 & 0.1111 & 0 & 0.1111 & $\mathbf{0 . 0 7 4 1}$ \\
10 & 0.1111 & 0.1111 & 0.1111 & 0.1111 & $\mathbf{0 . 0 7 4 1}$ & 0.1111 & 0.1111 & 0 & 0.1111 & 0 & 0.1481 \\
11 & 0.1250 & 0.1250 & 0.1250 & 0.1250 & 0.1250 & 0.1250 & 0.1250 & 0.1250 & 0 & 0 & 0 \\
\hline
\end{tabular}

\section{Experimental Design}

\subsection{Problem Sets}

In order to compare the performances of COIN against several comparator search heuristics, three well-known test problems were employed as shown in Table 5. The problem set 1, 2, and 3 represent small, medium, and large-sized problems respectively

\subsection{Comparison Heuristics}

The performances of the proposed COIN applied to MULB problems are compared against such a well-known multi-objective evolutionary as NSGA II. In addition, the

Table 5. Test problems

\begin{tabular}{llccc}
\hline & Problem Set & $\begin{array}{c}\text { Number of } \\
\text { Products }\end{array}$ & $\begin{array}{c}\text { Number } \\
\text { of Tasks }\end{array}$ & $\begin{array}{c}\text { Cycle } \\
\text { Time (sec) }\end{array}$ \\
\hline 1. & Thomopoulos[56] & 3 & 19 & 120 \\
2. & Kim[36] & 4 & 61 & 600 \\
3. & Arcus[57] & 5 & 111 & 10,000 \\
\hline
\end{tabular}

extended versions of COIN and NSGA II, i.e. MNSGA II and COIN-MA, are also evaluated.

\section{NSGA II}

The algorithm of NSGA II [46] can be stated as follows.

1) Create an initial parent population of size $N$ randomly.

2) Sort the population into several frontiers based on the fast non-dominated sorting algorithm.

3) Calculate a crowding distance measure for each solution.

4) Select the parent population into a mating pool based on the binary crowded tournament selection.

5) Apply crossover and mutation operators to create an offspring population of size $N$.

6) Combine the parent population with the offspring population and apply an elitist mechanism to the combined population of size $2 N$ obtain a new population of size $N$.

7) Repeat Step 2 until the terminating condition is met. MNSGA II

MNSGA II is a memetic version of NSGA II. Appro- 
priate local searches can additionally embed into several positions of the NSGA II's algorithm, i.e. after initial population, after crossover, and after mutation [52]. The number of places to apply local search has a direct effect on the quality of solution and computation time. Hence, if computation time needs to be saved, local search should be taken only at some specific steps in the algorithm of MA rather than at all possible steps. In this research, we choose to take local search after obtaining initial solution and after mutation since pilot experiments and our previous research [53] indicated that these two points were enough to find significantly improved solutions, pull the solutions out of the local optimal, and reduce computational time. The algorithm of MNSGA II can be stated as follows.

1) Create an initial parent population of size $N$ randomly.

2) Apply a local search to the initial parent population.

3) Sort the population into several frontiers based on the fast non-dominated sorting algorithm.

4) Calculate a crowding distance measure for each solution.

5) Select the parent population into a mating pool based on the binary crowded tournament selection.

6) Apply crossover and mutation operators to create an offspring population of size $N$.

7) Apply a local search to the offspring population.

8) Combine the parent population with the offspring population and apply an elitist mechanism to the combined population of size $2 N$ obtain a new population of size $N$.

9) Repeat Step 3 until the terminating condition is met.

Four local searches modified from Kumar and Singh [54] originally developed to solve travelling salesman problems by repeatedly exchanging edges of the tour until no improvement is attained are examined including Pairwise Interchange (PI), Insertion Procedures (IP), 2-Opt, and 3-Opt. Three criteria are used to test whether to accept a move that a local search heuristic creates a neighbour solution from the current solution as follows: (1) accept the new solution if $f_{1}(x)$ is descendent, (2) accept the new solution if $f_{1}(x)$ is the same and $f_{2}(x)$ is descendent; (2) accept the new solution if $f_{1}(x)$ is the same and $f_{3}(x)$ is descendent; or (3) accept the new solution if it dominates the current solution $\left(f_{1}(x)\right.$ is the same, and both $f_{2}(x)$ and $f_{3}(x)$ are descendent).

CNSGA II

In this heuristic, COIN is run for a certain number of generations. NSGA II then accepts the final solutions of COIN as its initial population and proceeds with its algorithm.

\section{COIN-MA}

In this heuristic, COIN is activated first for a certain number of generations. The final solutions obtained from
COIN are then fed into MNSGA II as an initial population.

\subsection{Comparison Metrics}

Three metrics are measured to evaluate the achievement of two common goals for comparison of multi-objective optimisation methods as recommended by Kumar and Singh [54]: 1) convergence to the Pareto-optimal set, and 2) maintenance of diversity in the solutions of Paretooptimal set. In addition, CPU time of each heuristic for achieving the final solutions is measured.

The convergence of the obtained Pareto-optimal solution towards a true Pareto-set $\left(A^{*}\right)$ is the difference between the obtained solution set and the true-Pareto set. Mathematically, it is defined as (9) and (10)

$$
\begin{gathered}
\text { convergence }(A)=\frac{\sum_{i=1}^{\left|A^{*}\right|} d t_{i}}{\left|A^{*}\right|} \\
d t_{i}=\min _{j=1}^{\left|A^{*}\right|} \sqrt{\sum_{k=1}^{2}\left[\frac{f_{k}(x)-f_{k}(y)}{f_{k}^{\max }-f_{k}^{\min }}\right]^{2}}
\end{gathered}
$$

where $\left|A^{*}\right|$ is the number of elements in set $A, d t_{i}$ is the Euclidean distance between non-dominated solution $i^{\text {th }}$ in the true-Pareto frontier $(y)$ and the obtained solution $(x), f_{k}^{\max }$ and $f_{k}^{\min }$ are maximum and minimum values of $k^{\text {th }}$ objective functions in the truePareto set respectively. For metric $A$, lower value indicates superiority of the solution set. When all solutions converge to Pareto-optimal frontier, this metric is zero indicating that the obtained solution set has all solutions in the true Pareto set. Since the true Pareto frontier is unknown, its approximation is needed. The approximated true Pareto-optimal frontier is the result of combining all final non-dominated solutions obtained from of all algorithms, applying Goldberg's Pareto ranking technique to the combined solutions, and the first frontier of the combined solutions is the approximated true Pareto-optimal frontier.

The second measure is a spread metric. This measure computes the distribution of the obtained Pareto-solutions by calculating a relative distance between consecutive solutions as shown in (11) and (12).

$$
\begin{gathered}
\operatorname{spread}(A)=\frac{s d_{f}+s d_{l}+\sum_{i=1}^{|A|-1}\left\|s d_{i}-s \bar{d}\right\|}{s d_{f}+s d_{i}+(|A|-1) s \bar{d}} \\
s d_{i}=\sqrt{\sum_{k=1}^{2}\left[\frac{f_{k}\left(x_{i}\right)-f_{k}\left(x_{i+1}\right)}{f_{k}^{\max }-f_{k}^{\min }}\right]^{2}}
\end{gathered}
$$

where $s d_{f}$ and $s d_{l}$ are the Euclidean distances between the extreme solutions and boundary solutions of the obtained Pareto-optimal, $|A|$ is the number of elements in the obtained-Pareto solutions, $s d_{i}$ is the Euclidian distance of between consecutive solutions in the obtained-Pareto solutions for $i=1,2, \ldots,|A|-1$, 
$s \bar{d}$ is the average Euclidean distance of $s d_{i}$, and the operator "II ||" means an absolute value. The value of this measure is zero for a uniform distribution, but it can be more than 1 when bad distribution is found.

The third measure is the ratio of non-dominated solutions $R_{N D S}\left(A_{j}\right)$ which indicates the coverage of one set over another. Let $A_{j}$ be a solution sets $(j=1,2, \ldots, J)$. For comparing each $J$ solution set $\left(A=A_{1} \cup A_{2} \ldots \cup\right.$ $A_{J}$ ) the ratio of non-dominated measure of the solution set $A_{j}$ to the $J$ solution sets is the ratio of solutions in $A_{j}$ that are not dominated by any other solution in $A$, which is defined as (13), where $y \prec x$ means the obtained solution $x$ is dominated by the true-Pareto solution $y$. The higher ratio indicates superiority of one solution set over another.

$$
R_{N D S}\left(A_{j}\right)=\frac{\left|A_{j}-\left\{x \in A_{j} \mid \exists y \in A: y<x\right\}\right|}{\left|A_{j}\right|}
$$

All algorithms are coded in Mathlab 7.0. The test platform is on Intel Core2 Duo $2.00 \mathrm{GHz}$ under Windows XP with 1.99 GB RAM. The CPU time of each heuristic is kept after the program is terminated.

\subsection{Parameter Settings}

To tune MOEA for the MULB problems, an experimental design [55] was employed to systematically conduct and investigate the effect of each parameter to the responses of each heuristic. Recommendations from the past researches, e.g. Kim et al. [36], Chutima and Pinkoompee [53], etc. were used as a starting point for parameter settings. Extensive pilot runs were conducted around the vicinities of the starting point. The selection for each parameter setting was based on quality and diversity of solutions. If neither quality nor diversity of solutions was significantly different for several settings of the parameter, the one with lowest CPU time was selected. Having done that, Table 6 shows the parameter settings found to be effective for each problem.

The process of finding appropriate local searches (LSs) for MNSGA II and COIN-MA for each problem set is worth mentioning. Four local searches that gave good performances from previous research [53] were investigated, i.e. Pairwise Interchange (PI), Insertion Procedures (IP), 2-Opt, and 3-Opt. Although LS can be located on 3 different places in MA, pilot runs indicated that putting LS after crossover did not help MA improve its performances. Therefore, LSs were placed only after initial population and after mutation for MNSGA II and after mutation for COIN-MA. Full factorial experiments were conducted to test the performances of LSs on each problem with 2 replicates. The number of experiment runs for each problem of MNSGA II and COIN-MA is $4 * 4 * 2=32$ and $4 * 2=8$ respectively. In total the number of runs is 120. ANOVA and Tukey's multiple range test were conducted to test significant different at 0.05 level.

Table 6. Parameter settings for each heuristic

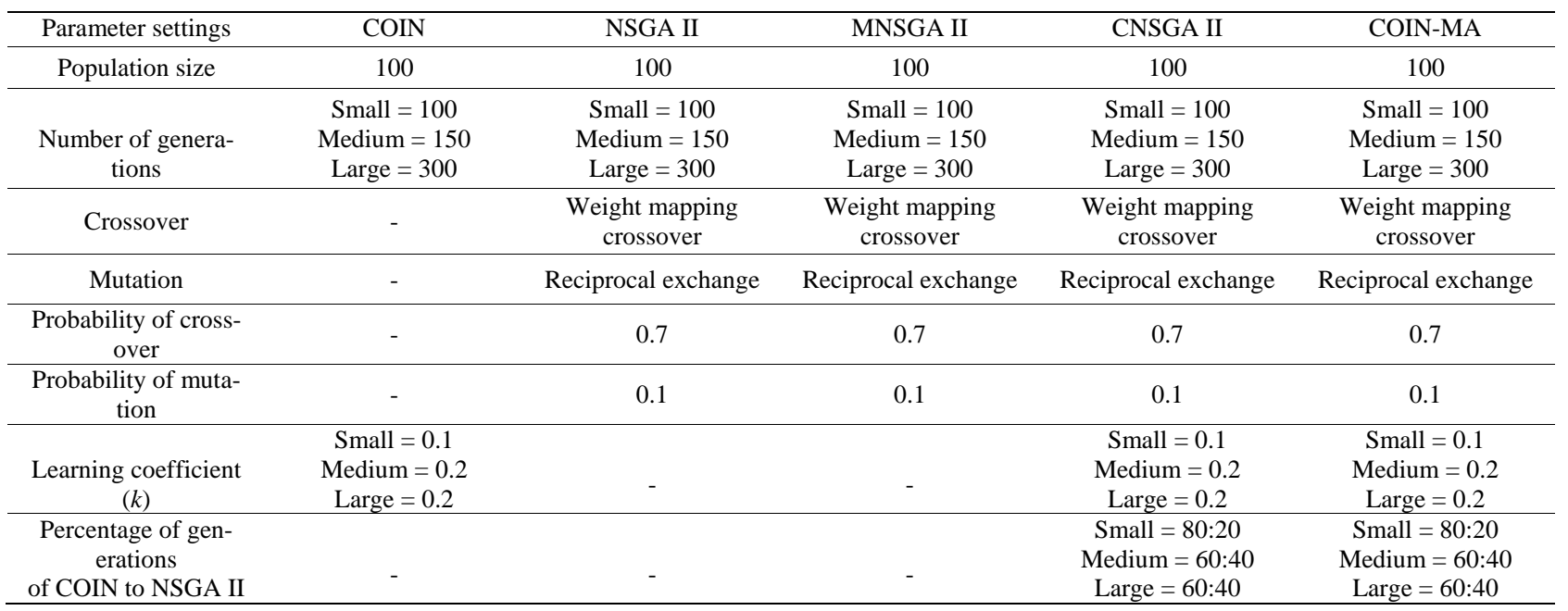

Table 7. Appropriate local searches

\begin{tabular}{cccc}
\hline Problem set & & MNSGA II & COIN-MA \\
\cline { 2 - 4 } & LS after & LS after \\
mutation & PI & LS after mutation \\
2 & initial population & IP-Opt & PI \\
3 & PI & IP & PI \\
\hline
\end{tabular}


The LSs appearing in Table 7 were those that performed best with respect to solution quality, diversity, and CPU time. It is apparent that best LS combination for MNSGA II and COIN-MA depends on the problem set. However, for MNSGA II the combination of IP (LS used after initial population) and PI (LS used after mutation) appear more often than the other. For COIN-MA, IP seems to give better performances for small- and medium-sized problems; whereas, PI performed better than the others for large-sized problems. As a result, these settings were used for MNSGA II and COIN-MA in relative performance comparison.

\section{Experimental Results}

The behaviour of COIN was demonstrated with the 61 tasks' problem as shown in Figure 6. At the beginning (generation 1), a number of rather poor feasible solutions were created. As the number of generations increased, better solutions were found as observed from the moving downward trend to the left of the Pareto fronts. It was noticeable that not much improvement was gained in the first 20 generations. A leaped gain was noticeable from generations 20 to 30 . However, the improvement was less and less after that and the Pareto front remained the same after generation 100 .

The behaviour of CNSGA II (COIN plus NSGA II) and COIN-MA (COIN plus MNSGA II) were demonstrated in Figure 7 and Figure 8. Both algorithms allowed COIN to run for 150 generations and the final solutions of COIN were considered as initial solutions of NSGA II and MNSGA II. Significant improvement was found after COIN was terminated and marginal gains from its previous solutions were obtained at the end of both algorithms. In other words, NSGA II (in CNSGA II) and MNSGA II (in COIN-MA) cannot provide much improvement to the final solutions of COIN.

For the small-sized problem (19 tasks), Table 8 showed that all algorithms gave the same number of workstations. NSGA II performed worst comparing with the others. By adding appropriate local search to NSGA II, its memetic version (MNSGA II) gained significant performance improvement. Although MNSGA II obtained the best spread metric, it was dominated by COIN, CNSGA II, and COIN-MA (Figure 9). These three algorithms obtained the same best Pareto front which can be seen from their ratio of non-dominated solution $\left(R_{N D S}=\right.$ 1) in Table 8.

For the medium-sized problem (61 tasks), COIN-MA obtained highest $R_{N D S}$, followed by CNSGA II, whereas the other algorithms have $R_{N D S}=0$ (Table 8). This was confirmed by Figure 10 meaning that some solutions of COIN-MA and CNSGA II were located in the Pareto front. MNSGA II outperformed NSGA II, but it was dominated by COIN. A big gap between the front of NSGA II and the

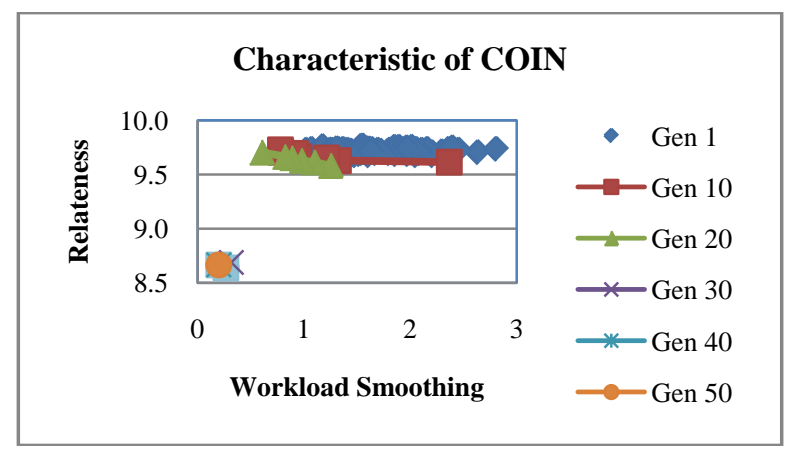

Figure 6. Characteristic of COIN

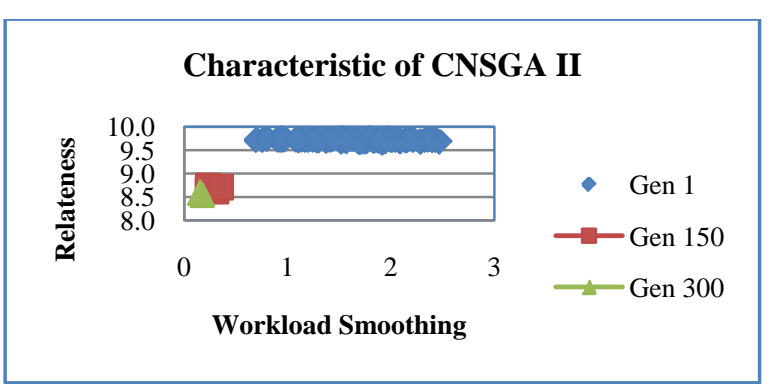

Figure 7. Characteristic of CNSGA II.

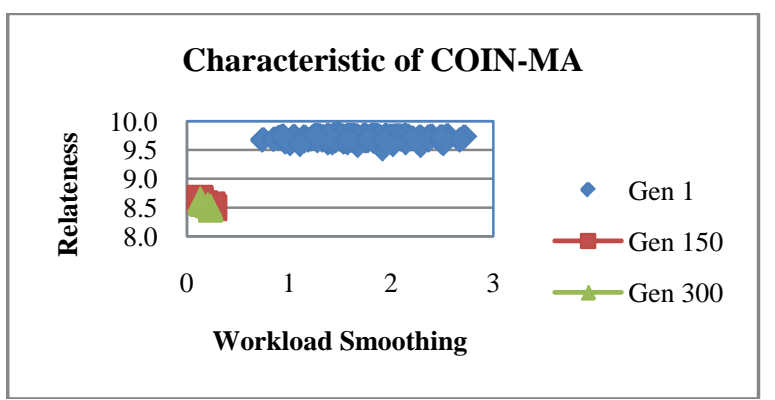

Figure 8. Characteristic of COIN-MA

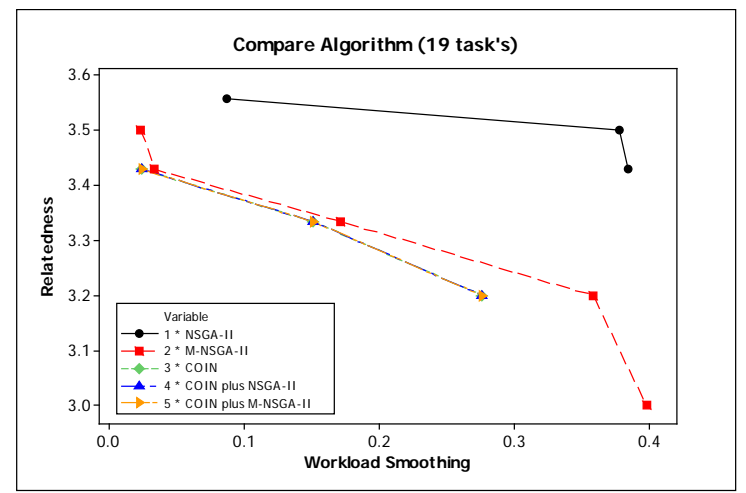

Figure 9. Pareto front of each algorithm (19 tasks)

fronts of three good performers (COIN, CNSGA II, and COIN-MA) was noticed indicating significant gains from using these three algorithms. 
Table 8. Performance comparison

\begin{tabular}{|c|c|c|c|c|c|c|}
\hline Problem set & $\begin{array}{l}\text { Performance Meas- } \\
\text { ure }\end{array}$ & NSGA II & COIN & MNSGA II & CNSGA II & COIN-MA \\
\hline \multirow{5}{*}{1} & $\begin{array}{c}\text { Number of work- } \\
\text { stations }\end{array}$ & 4 & 4 & 4 & 4 & 4 \\
\hline & Convergence & 0.4381 & 0.1317 & 0.0603 & 0.1317 & 0.1317 \\
\hline & Spread & 0.6557 & 0.5390 & 0.4948 & 0.5390 & 0.5390 \\
\hline & $R_{N D S}$ & 0.0000 & 1.0000 & 0.4000 & 1.0000 & 1.0000 \\
\hline & CPU Time (min) & 6 & 3 & 13 & 4 & 7 \\
\hline \multirow{5}{*}{2} & $\begin{array}{c}\text { Number of work- } \\
\text { stations }\end{array}$ & 10 & 9 & 10 & 9 & 9 \\
\hline & Convergence & 0.9951 & 0.8966 & 0.4419 & 0.3058 & 0.0710 \\
\hline & Spread & 0.4504 & 0.4945 & 0.8038 & 0.5514 & 0.4271 \\
\hline & $R_{N D S}$ & 0.0000 & 0.0000 & 0.0000 & 0.5000 & 0.6250 \\
\hline & CPU Time (min) & 55 & 11 & 86 & 19 & 25 \\
\hline \multirow{5}{*}{3} & $\begin{array}{l}\text { Number of work- } \\
\text { stations }\end{array}$ & 16 & 16 & 16 & 15 & 15 \\
\hline & Convergence & 1.0000 & 0.9907 & 0.8645 & 0.4100 & 0.0000 \\
\hline & Spread & 0.7479 & 0.6951 & 0.4882 & 0.7211 & 0.6643 \\
\hline & $\mathrm{R}_{\mathrm{NDS}}$ & 0.0000 & 0.0000 & 0.0000 & 0.0000 & 1.0000 \\
\hline & CPU Time (min) & 478 & 20 & 1089 & 32 & 44 \\
\hline
\end{tabular}

For the large-sized problem (111 tasks), once again, COIN-MA performed best and NSGA II was ranked last (Figure 11). COIN outperformed NSGA and NSGA II. The performance of COIN was improved significantly with the cooperation of NSGA II (CNSGA II) and MNSGA II (COIN-MA). COIN-MA dominated all algorithms and, from Table 8, its solutions were all in the Pareto front $\left(R_{N D S}=1\right)$.

In terms of CPU time (Table 8), COIN used the lowest time to achieve the final solutions followed by CNSGA II,

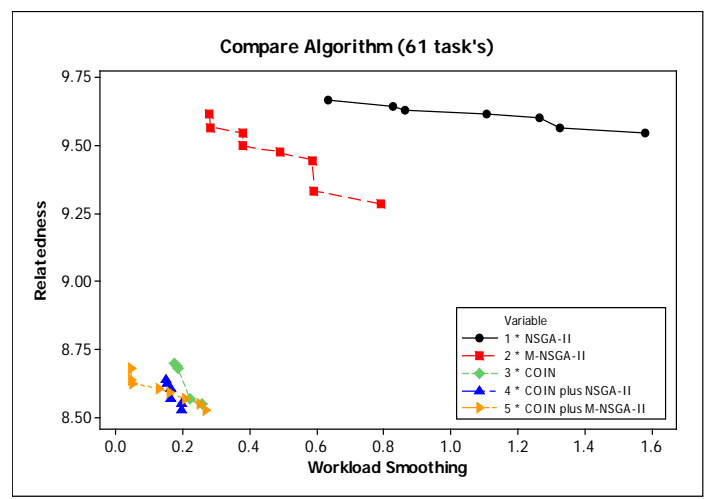

Figure 10. Pareto front of each algorithm (61 tasks)
COIN-MA, NSGA II, and MNSGA II. As a result, COIN can be considered as a fast and smart algorithm since it can obtain good solutions very fast. It can be used as a good benchmark for other algorithms. In addition, if the good Pareto front needs to be discovered within a limited CPU time, COIN-MA is recommended as an outstanding alternative.

\section{Conclusions}

This paper presents a novel evolutionary algorithm

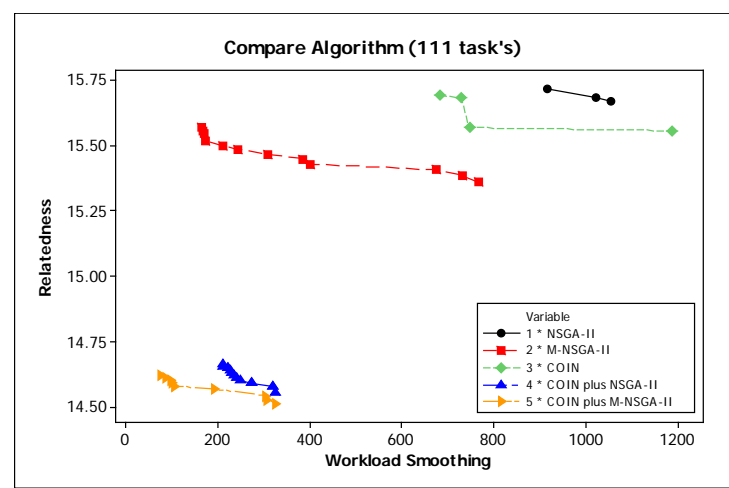

Figure 11. Pareto front of each algorithm (111 tasks) 
namely combinatorial optimisation with coincidence algorithm (COIN) and its variances. The algorithms are applied to solve Type I problems of MMUALBP in a just-in-time production environment. COIN recognises the positive knowledge appearing in the order pairs of the good solution by giving a marginal reward (increased probability) to its related element of the joint probability matrix. In contrast, the negative knowledge found in the order pairs of the bad solution, which is often remiss in most algorithms, is also utilised in COIN (reduced probability) to prevent undesired solutions coincidentally found in this generation to be recurring in the next generation. The performances of COIN and its variances are evaluated on three objectives, i.e. minimum number workstations, minimum work relatedness, and minimum workload smoothness. Among these three, minimum number of workstations is dominated resulting in only the solutions with the same minimum number of workstations being considered and can be located on the first Pareto front. Experimental results indicate clearly that COIN outperforms the well-known NSGA II in all aspects. As a result, COIN can be considered as a new alternative benchmarking algorithm for MMUALBP. The COIN's variances (CNSGA II and COIN-MA) show significantly better performances than COIN, NSGA II, and MNSGA II. Although COIN-MA marginally uses more CPU time than CNSGA II, the other performances of COIN-MA are better than CNSGA. As a result, if we need to find an algorithm to search for an optimal Pareto front for MMUALBP, COIN-MA is recommended.

\section{REFERENCES}

[1] N. Boysen, M. Fliedner and A. Scholl, “Assembly Line Balancing: Which Model to Use When?” International Journal of Production Economics, Vol. 111, No. 2, 2008, pp. 509-528.

[2] R. J. Schonberger, “Japanese Manufacturing Techniques: Nine Hidden Lessons in Simplicity," Free Press, New York, 1982, pp. 140-141.

[3] J. Miltenburg, "U-Shaped Production Lines: A Review of Theory and Practice," International Journal of Production Economics, Vol. 70, No. 3, 2001, pp. 201-214.

[4] Y. Monden, “Toyota Production System," 2nd Edition, Industrial Engineering Press, Institute of Industrial Engineering, Norcross, 1993.

[5] G. J. Miltenburg and J. Wijngaard, "The U-Line Balancing Problem,” Management Science, Vol. 40, No. 10, 1994, pp. 1378-1388.

[6] C. H. Cheng, G. J. Miltenburg and J. Motwani, "The Effect of Straight- and U-Shaped Lines on Quality," IEEE Transactions on Engineering Management, Vol. 47, No. 3, 2000, pp. 321-334.

[7] G. J. Miltenburg, "The Effect of Breakdowns on U-Shaped
Production Lines,” International Journal of Production Research, Vol. 38, No. 2, 2000, pp. 353-364.

[8] J. Miltenburg, "One-Piece Flow Manufacturing on UShaped Production Lines: A Tutorial,” IIE Transactions, Vol. 33, No. 4, 2001, pp. 303-321.

[9] Y. Kara, U. Ozcan and A. Peker, "Balancing and Sequencing Mixed-Model just-in-Time U-Lines with Multiple Objectives," Applied Mathematics and Computation, Vol. 184, No. 2, 2007, pp. 566-588.

[10] M. E. Salveson, “The Assembly Line Balancing Problem,” The Journal of Industrial Engineering, Vol. 6, No. 3, 1955, pp. 18-25.

[11] I. Baybars, "A Survey of Exact Algorithms for the Simple Assembly Line Balancing Problem,” Management Science, Vol. 32, No. 8, 1986, pp. 909-932.

[12] S. Ghosh and R. J. Gagnon, “A Comprehensive Literature Review and Analysis of the Design, Balancing and Scheduling of Assembly Systems," International Journal of Production Research, Vol. 27, No. 4, 1989, pp. 637-670.

[13] E. Erel and S. C. Sarin, "A Survey of the Assembly Line Balancing Procedures,” Production Planning and Control, Vol. 9, No. 5, 1998, pp. 414-434.

[14] C. Becker and A. Scholl, "A Survey on Problems and Methods in Generalized Assembly Line Balancing," European Journal of Operational Research, Vol. 168, No. 3, 2006, pp. 694-715.

[15] N. Boysen and M. Fliedner, "A Versatile Algorithm for Assembly Line Balancing,” European Journal of Operational Research, Vol. 184, No. 1, 2008, pp. 39-56.

[16] D. Sparling and J. Miltenburg, “The Mixed-Model U-Line Balancing Problem,” International Journal of Production Research, Vol. 36, No. 2, 1998, pp. 485-501.

[17] G. J. Miltenburg, "Balancing U-Lines in a Multiple ULine Facility," European Journal of Operational Research, Vol. 109, No. 1, 1998, pp. 1-23.

[18] T. L. Urban, “Optimal Balancing of U-Shaped Assembly Lines,” Management Science, Vol. 44, No. 5, 1998, pp. 738-741.

[19] D. A. Ajenblit and R. L. Wainwright, “Applying Genetic Algorithms to the U-Shaped Assembly Line Balancing Problem," Proceedings of the 1998 IEEE International Conference on Evolutionary Computation, Alaska, 1998, pp. 96-101.

[20] A. School and R. Klein, "ULINO: Optimally Balancing U-Shaped JIT Assembly Lines," International Journal of Production Research, Vol. 37, No. 4, 1999, pp. 721-736.

[21] E. Erel, I. Sabuncuoglu and B. A. Aksu, "Balancing of U-Type Assembly Systems Using Simulated Annealing," International Journal of Production Research, Vol. 39, No. 13, 2001, pp. 3003-3015.

[22] G. R. Aase, M. J. Schniederjans and J. R. Olson, “U-OPT: An Analysis of Exact U-Shaped Line Balancing Procedures," International Journal of Production Research, Vol. 41, No. 17, 2003, pp. 4185-4210. 
[23] G. R. Aase, J. R. Olson and M. J. Schniederjans, "UShaped Assembly Line Layouts and their Impact on Labor Productivity: An Experimental Study,” European Journal of Operational Research, Vol. 156, No. 3, 2004, pp. 698711.

[24] U. Martinez and W. S. Duff, "Heuristic Approaches to Solve the U-Shaped Line Balancing Problem Augmented by Genetic Algorithms," Proceedings of the 2004 Systems and Information Engineering Design Symposium, Charlottesville, 16 April 2004, pp. 287-293.

[25] J. Balakrishnan, C.-H. Cheng, K.-C. Ho and K. K. Yang, "The Application of Single-Pass Heuristics for U-Lines," Journal of Manufacturing Systems, Vol. 28, No. 1, 2009, pp. 28-40.

[26] H. Gokcen and K. Agpak, “A Goal Programming Approach to Simple U-Line Balancing Problem,” European Journal of Operational Research, Vol. 171, No. 2, 2006, pp. 577-585.

[27] T. L. Urban and W.-C. Chiang, “An Optimal Piecewise-Linear Program for the U-Line Balancing Problem with Stochastic Task Times," European Journal of Operational Research, Vol. 168, No. 3, 2006, pp. 771-782.

[28] W.-C. Chiang and T. L. Urban, "The Stochastic U-Line Balancing Problem: A Heuristic Procedure,” European Journal of Operational Research, Vol. 175, No. 3, 2006, pp. 1767-1781.

[29] Y. Kara, T. Paksoy and C. T. Chang, "Binary Fuzzy Goal Programming Approach to Single Model Straight and U-Shaped Assembly Line Balancing," European Journal of Operational Research, Vol. 195, No. 2, 2009, pp. 335347. A. L. Arcus, "COMSOAL: A Computer Method of Sequencing Operations for Assembly Lines," International Journal of Production Research, Vol. 4, No. 4, 1965, pp. 259-277.

[30] A. Baykasoglu, "Multi-Rule Multi-Objective Simulated Annealing Algorithm for Straight and U Type Assembly Line Balancing Problems," Journal of Intelligent Manufacturing, Vol. 17, No. 2, 2006, pp. 217-232.

[31] R. K. Hwang, H. Katayama and M. Gen, "U-Shaped Assembly Line Balancing Problem with Genetic Algorithm," International Journal of Production Research, Vol. 46, No. 16, 2008, pp. 4637-4649.

[32] R. K. Hwang and H. Katayama, "A Multi-Decision Genetic Approach for Workload Balancing of Mixed-Model U-Shaped Assembly Line Systems,” International Journal of Production Research, Vol. 47, No. 14, 2009, pp. 37973822.

[33] A. Baykasoglu and T. Dereli, "Simple and U-Type Assembly Line Balancing by Using an Ant Colony Based Algorithm," Mathematical and Computational Applications, Vol. 14, No. 1, 2009, pp. 1-12.

[34] G. J. Miltenburg, "Balancing and Scheduling Mixed-Model U-Shaped Production Lines," International Journal of Flexible Manufacturing Systems, Vol. 14, No. 2, 2002, pp. 119-151.
[35] Y. K. Kim, S. J. Kim and J. Y. Kim, "Balancing and Sequencing Mixed-Model U-Lines with a Co-Evolutionary Algorithm,” Production Planning and Control, Vol. 11, No. 8, 2000, pp. 754-764.

[36] Y. K. Kim, J. Y. Kim and Y. Kim, “An Endosymbiotic Evolutionary Algorithm for the Integration of Balancing and Sequencing in Mixed-Model U-Lines," European Journal of Operational Research, Vol. 168, No. 3, 2006, pp. 838-852.

[37] S. Agrawal and M. K. Tiwari, "A Collaborative Ant Colony Algorithm to Stochastic Mixed-Model U-Shaped Disassembly Line Balancing and Sequencing Problem,” International Journal of Production Research, Vol. 46, No. 6, 2008, pp. 1405-1429.

[38] I. Sabuncuoglu, E. Erel and A. Alp, “Ant Colony Optimization for the Single Model U-Type Assembly Line Balancing Problem,” International Journal of Production Economics, Vol. 120, No. 2, 2009, pp. 287-300.

[39] Y. Kara, U. Ozcan and A. Peker, "An Approach for Balancing and Sequencing Mixed-Model JIT U-Lines,” International Journal of Advanced Manufacturing Technology, Vol. 32, No. 11-12, 2007, pp. 1218-1231.

[40] Y. Kara, "Line Balancing and Model Sequencing to Reduce Work Overload in Mixed-Model U-Line Production Environments,” Engineering Optimization, Vol. 40, No. 7, 2008, pp. 669-684.

[41] A. Konak, D. W. Coit and A. E. Smith, "Multi-Objective Optimization Using Genetic Algorithms: A Tutorial,” Reliability Engineering \& System Safety, Vol. 91, No. 9, 2006, pp. 992-1007.

[42] C. A. C. Coello, D. A. Veldhuizen and G. B. Lamont, "Evolutionary Algorithms for Solving Multi-Objective Problems,” Kluwer Academic Publishers, Dordrecht, 2002.

[43] E. Zitzler and L. Thiele, "Multiobjective Evolutionary Algorithms: A Comparative Case Study and the Strength Pareto Approach," IEEE Transactions on Evolutionary Computation, Vol. 3, No. 4, 1999, pp. 257-271.

[44] C. M. Fonseca and P. J. Fleming, “Genetic Algorithms for Multiobjective Optimization: Formulation, Discussion and Generalization,” Proceedings of 5th International Conference on Genetic Algorithm, Urbana, June 1993, pp. 416423.

[45] C. M. Fonseca and P. J. Fleming, “An overview of Evolutionary Algorithms in Multiobjective Optimization,” Evolutionary Computation, Vol. 3, No. 1, 1995, pp. 1-16.

[46] K. Deb, A. Pratap, S. Agarwal and T. Meyarivan, "A Fast and Elitist Multiobjective Genetic Algorithm: NSGA II," IEEE Transactions on Evolutionary Computation, Vol. 6, No. 2, 2002, pp. 182-197.

[47] D. Corne, M. Dorigo and F. Glover, "New Ideas in Optimization,” McGraw-Hill, London, 1999.

[48] W. Wattanapornprom, P. Olanviwitchai, P. Chutima and P. Chongsatitvatana, "Multi-Objective Combinatorial Op- 
timisation with Coincidence Algorithm,” Proceedings of IEEE Congress on Evolutionary Computation, Norway, 11 February 2009, pp. 1675-1682.

[49] J. R. Jackson, “A Computing Procedure for a Line Balancing Problem,” Management Science, Vol. 2, No. 3, 1956, pp. 261-271.

[50] D. E. Goldberg, "Genetic Algorithms in Search, Optimization, and Machine Learning,” Addison-Wesley, Boston, 1989.

[51] J. Horn, N. Nafpliotis and D. E. Goldberg, “A Niched Pareto Genetic Algorithm for Multiobjective Optimization," Proceedings of the First IEEE Conference on Evolutionary Computation, IEEE World Congress on Computational Intelligence, Orlando, 27-29 June 1994.

[52] P. Lacomme, C. Prins and M. Sevaux, "A Genetic Algorithm for a Bi-Objective Capacitated ARC Routing Problem,” Computer \& Operations Research, Vol. 33, No. 12, 2006, pp. 3473-3493.

[53] P. Chutima and P. Pinkoompee, “An Investigation of Lo- cal Searches in Memetic Algorithms for Multi-Objective Sequencing Problems on Mixed-Model Assembly Lines,” Proceedings of Computers and Industrial Engineering, Beijing, 31 October-2 November 2008.

[54] R. Kumar and P. K. Singh, "Pareto Evolutionary Algorithm Hybridized with Local Search for Bi-Objective TSP," Studies in Computational Intelligence (Hybrid Evolutionary Algorithms), Springer, Berlin/Heidelberg, Vol. 75, 2007, pp. 361-398.

[55] D. C. Montomery, "Design and Analysis of Experiments," John Wiley \& Sons, Inc., Hoboken, 2009.

[56] N. T. Thomopoulos, "Mixed Model Line Balancing with Smoothed Station Assignment," Management Science, Vol. 14, No. 2, 1970, pp. B59-B75.

[57] A. L. Arcus, "COMSOAL: A Computer Method of Sequencing Operations for Assembly Lines,” International Journal of Production Research, Vol. 4, No. 4, 1965, pp. 259-277. 